\title{
ASTRONOMICAL DATING AND STATISTICAL ANALYSIS OF ANCIENT CHINESE ECLIPSE DATA
}

\author{
KEVIN D. PANG \\ Fax: USA code + 818952 1371, E-mail: KevinDPang@aol.com \\ KEVIN K. YAU \\ Jet Propulsion Laboratory $M / S$ 230-101, 4800 Oak Grove Dr., Pasadena, California, \\ 91109, USA \\ AND \\ HUNG-HSIANG CHOU \\ Dept. of East Asian Languages and Cultures, Univ. California, Los Angeles 90024, \\ $U S A$
}

\begin{abstract}
All 13 Shang dynasty oracle bone eclipse records have been uniquely matched to 6 solar and 7 lunar eclipses in the 14-12th centuries B.C. The King Zhong Kang 5th year autumnal (Oct. 16, 1876 B.C.) and King Yu 3rd year "double sunset" (Sept. 24, 1912 B.C.) eclipses confirm the accuracy of the revised Bamboo Annals Xia dynasty chronology (Nivision and Pang, Early China 15, 1990, 87). The eclipse dates are plotted against the number of generations before 841 B.C. (earliest accurate date), the respective kings ruled. The curve of benefit has both the strengths of verified royal genealogy - continuity - and eclipse dating - accuracy. It is $99 \%$ accurate, and can be confidently used as a foundation for building a detailed absolute chronology for the Xia, Shang and Zhou dynasties, an important project in China's new 5-Year Plan (Song, Sci. Tech. Daily, May 17, 1996; Newsweek, July 7,1997$)$.
\end{abstract}

\section{Introduction}

Before the Shang oracle bones (discovered in an apothecary in 1899) were analyzed some scholars considered the dynasty to be legendary. It has now been proven that records had been accurately passed down to historian Sima Qian (91 B.C.) and the authors of the Bamboo Annals (229 B.C.). The Kinglist, compiled from them, is identical to that of Sima Qian and the Bamboo Annals. The genealogy of the Xia kings has not yet been directly verified by archaeological finds, but the absolute chronology of early Xia from King Yu to Zhong Kang, as recorded in the Bamboo Annals, has been confirmed by astronomical evidence (Nivison and Pang, 1990; Pang and Yau, 1996).

Astronomical dating involves retroactively computing and matching the circumstances of a celestial phenomenon observed by ancient people, but recorded without precise date. We can combine the strength of royal genealogy - continuity - with that of astronomical dating - accuracy - by correlating them. Our process is similar to stretching and contracting an approximately ruled and slightly flexible (genealogical) scale here and there to line up selected ticks with independent benchmarks (eclipse dates). We use solar, lunar and planetary ephemerides, e.g., JPL DE102, to compute past positions. Eclipse computing programs, with the latest lunar acceleration rate and rotational history of the Earth as input, are also used. Computer search can help us find the correct match. In the next six sections we will give specific examples of eclipse dating, beginning with fascinating records of "double dawn" eclipses at Zheng. 


\section{Double Dawns at Zheng and the First Year of King Yi of the Western Zhou Dynasty}

The Bamboo Annals, entombed in 299 B.C. and unearthed in A.D. 281, states that "in the first month of spring in the first year of King Yi of Western Zhou, he ascended the throne, the day dawned twice at Zheng $\left(34.5^{\circ} \mathrm{N}, 109.8^{\circ} \mathrm{E}\right)$ " (now Hua District, Shaanxi). Kaiyuan zhanjing, compiled by Indian astronomer Gautama Siddharta (A.D. 724), cites this passage and adds that "in the 2nd (actually 12th) year of Sheng Ping reign period of King Shang - (actually Early Liang King Zhang Tian Xi) the day (also) began twice at Zheng (italic ours)." These events have been uniquely matched with April 21, 899 B.C. and April 4, A.D. 368 sunrise eclipses at Zheng (Pang et al., 1995). The first year of King Yi, heretofore known only to be between 966 and 895 B.C., can now be firmly fixed at 899 B.C. We will discuss the dating of Shang dynasty oracle bone eclipse records in the next three sections.

\section{Shang Dynasty Period One Oracle Bone Lunar and Solar Eclipse Records Dated}

Five Period I (King Wu Ding) lunar eclipse records are listed below in the order of decreasing relative ages, ranked according to evolutionary advances in the style of calligraphy of the characters used (Takashima, 1998):

\begin{aligned} Cyclic Day Stated & $\frac{(\text { Modulo 60) }}{31} \\$\cline { 2 - 2 } 57 & gena-wu \\ 20 & gui-usei \\ 22 & yi-you \\ 9 & reri-shen \end{aligned}

The 60-day cycle has been in continous use from time immemorial. Matching eclipse dates should range $<59$ years (Wu Ding's reign). Assuming that the Shang day was either "Egyptian" (dawn to dawn) or "Roman" (midnight to midnight) previous researchers could not find five computed eclipses that match all conditions (Chang, 1980). However the ancient Chinese day, unlike either, began at $\sim 3$ a.m. instead. Events occurring between midnight and dawn had a $85 \%$ probability of beeing recorded with the old date, and $15 \%$ with the new (King, 1981).

Armed with this new knowledge and a delta T, derived from the Period I (June 5, 1302 B.C.) Shang oracle bone solar eclipse record, that states "three flames ate the Sun, big stars were seen," Pang et al. $(1989,1995)$ successfully matched all five records. As they all occurred between midnight and dawn the odds are that one would carry the new date, and the rest - the old. This turns out to be exactly the case: The 1322 B.C. eclipse is the odd one. Our dates span only 44 years, and are in the same order as Takashima's, except two of the latest inscriptions are transposed. Separated by less than four years they are simply too close together to be ranked by calligraphic change. Having successfully matched all Period I eclipse records we now go on to date the Period IV eclipse records.

\section{Shang Dynasty Period Four Oracle Bone Solar and Lunar Eclipse Records Dated}

Of the Period IV (Kings Wu Yi and Wen Ding) solar eclipse records listed by Zhang (1975) and Xi (1984), inscription Nos. 33694 and 33703 are only inquiries about whether an eclipse will occur. Unless otherwise noted we use Guo Moruo's $\mathrm{He}-j i$ index system. Since these are not observational records they are not useful to us $(\mathrm{Hu}, 1986)$.

The relevant contents of the valid records are: No. 33696 - "Day yi-si (42nd day)...Sun has zhi...reported to Shang Jia that cvening-night, nine oxen sacrificed." No. 33698 - "Day geng-chen (17th day)...Sun has zhi...reported to He and Father Ding...nine oxen sacrificed..." No. 33699 "Day $u n-z i$ (25th day)...Sun has zhi...reported to He." This one has been misdeciphered as day wu-shen (45th day) before (Yao and Xiao, 1988). No. 33700 - "Inquiry on day yi-chou (2nd day): 'Will Sun have zhi?' (verification) 'It indeed had zhi.' " No. 33710 - "Day xin-si (18th day)...Sun (has) zhi...reported to Father Ding." The large number of oxen sacrificed, and the prompt reporting to ancestors or deity, betray the Shang's great concern about the changes that had occured on the Sun. 
These inscriptions use the phrase "has $z h \imath^{\prime}$ rather than the "has shi (been eaten)," typical in Period I. The best explanation for this is that primitive Chinese characters that sound alike were used interchangeably $(\mathrm{Xi}, 1984)$. The "borrowed usage" of words with a similar sound is very common in Shang and Zhou inscriptions. Of the 15 Shang eclipse inscriptions with a cyclic date, eight use the term "shl" and seven use "zhi." The even split can be considered as a proof of dual usage until at least Period IV. We have found further proof of the interchangeability of the two terms by considering the context where $z h i$ appears in various sentences.

First, zhi seems to refer to a. foreseeable change on the Sun, as No. 33700 had shown. The anticipatable time could be two days, as No. 33703 also states - "Divination on day ren-ji (49th day): 'Will the Sun have zhi on day jia-yin (51st day)?' " Once some believed that $z h i$ refers to sunspots. However in 1972 an ox scapula (Tun-nan No. 726) was unearthed from Anyang $\left(36^{\circ} \mathrm{N}\right.$, $114^{\circ} \mathrm{E}$ ), inscribed with "Inquiry on day ren-yin (39th day): 'The Moon has zhi..." (Xi, 1984). Since the Moon does not have transient spots we are forced to conclude that zhi refers to a foreseeable change that can happen to both the Sun and Moon, and by analogy with shi, clearly also means "been eclipsed." Having established that these are bona fide eclipse records Pang et al. (1995) dated them as follows:

\begin{tabular}{|c|c|c|c|c|c|}
\hline Inscription & & Cyclic & Eclipse & & Local Time \\
\hline No. & Day No. & Day Name & Date (B.C.) & Magnitude & Hour Min. \\
\hline 33700 & 2 & $\overline{y i-c h o u}$ & $5 / 6 / 1226$ & 0.78 & $\overline{11} \overline{44}$ \\
\hline 33698 & 17 & geng-chen & $10 / 21 / 1198$ & 0.73 & 1740 \\
\hline 33710 & 18 & xin-si & $6 / 7 / 1172$ & 0.87 & $06 \quad 17$ \\
\hline 33699 & 25 & $w u-z i$ & $6 / 27 / 1163$ & 0.43 & 1911 \\
\hline 33696 & 42 & $y i-s i$ & $10 / 31 / 1161$ & 0.86 & $06 \quad 29$ \\
\hline
\end{tabular}

Notes
All 5 records use the phrase
"Ri you (Sun has) zhi." Note
both "has" and "zhl" differ
from the forms used in Period
I, as noted in previous table.

Our dates agree with Zhang's (1975), except for No. 33699. He matched the misdeciphered day 45 wu-shen record with May 7, 1161 B.C. solar eclipse, which was not visible from China. The Period IV "Moon has $z h l$ " record can be matched with the July 2-3, 1173 B.C. lunar eclipse. Some have suggested that the phrase "has $z h i$ " refers to red Sun or Moon. However it is highly unlikely that the reddening atmospheric processes can randomly generate 20 such sightings in 65 years, with six coinciding with an eclipse day or night in a Saros cycle, a unique and highly systematic time series trend. Actuary tables put the odds of six such coincidences out of 20 at 1000 to 1 (Nolan, 1975)! The frequency of red Sun and Moon sightings is estimated from a count of 145 such events, reported between A.D. 1030 and 1500 (Gujin Tushu Jicheng, a 1726 compendium of earlier events). One can also get a good feel for the degree of difficulty in matching just one solar eclipse record within 40 years in the next section.

\section{Shang Dynasty Period Three Oracle Bone Lunar Eclipse Record Dated}

The only oracle bone eclipse record from Period III (Kings Lin Xin and Kang Ding), Zhui-he No. 465, states - "Divination on day jia-yin (51st day): 'There was an eclipse (shi). Should it be reported?' "It does not say solar or lunar, so it would seem easy to match. However the choices proved to be very limited. First, we must choose in the 52 years between the latest (1278 B.C.) Period I eclipse and the earliest (1226 B.C.) Period IV eclipse. Second, it should be closer to 1226 B.C., as the Period II king $\mathrm{Zu}$ Jia reigned for 33 years. No solar eclipse visible from China, occurred on day jia-yin from the 1250's through the 1220's B.C., but the March 20, 1242 B.C. lunar eclipse fits perfectly. Viewed from Anyang the Moon rose eclipsed that evening. Having successfully matched all Shang oracle bone eclipse records with at least a cyclic date, we go on to date the two Xia dynasty solar eclipse records.

\section{The Book of $X i a$ Solar Eclipse and the Fifth Year of King Zhong Kang of the Xia Dynasty}

The world famous King Zhong Kang solar eclipse is based on three sources: (1) The "Yin cheng (Punitive Expedition of Prince Yin)" chapter of Shujing (Book of Documents) states that "...on the first day of the last month of autumn, the chen was not harmonious in (lunar mansion) Fang, the blind beat drums, junior officers galloped, people ran around..." Astronomer Xihe was punished 
for failing to note the eclipse. (2) The Zuo zhuan's citation of the Xia shu or Book of Xia (Duke Zhao, Year 17) is identical, except that is says the eclipse was in the first month of summer. (3) The Bamboo Annals sides with the Book of Documents, and puts it in King Zhong Kang's fifth year.

An extensive analysis of the Zuo zhuan passage (Pang, 1990) is summarized as follows: (1) The meaning of "chen" is given in Zuo zhuan, Duke Zhao, Year 7 (535 B.C.) - "Chen is the meeting of the Sun and Moon." Before they understood what causes eclipses the Chinese thought that they were due to "inharmonious" meetings of the Sun and Moon. (2) The month of the eclipse in Zuo zhuan is wrong, because if a solar eclipse occurred in Fang it could not possibly have been the first month of summer. During this month the Sun was in Bi, a third of the sky away! Only during the last month of autumn could the Sun have been in Fang, which is only $5^{\circ}$ wide (Chen, 1988).

The Bamboo Annals states that "a solar eclipse occurred on the first day of the ninth lunar month, day geng-xu, autumn, in the fifth year of King Zhong Kang, and the Prince of Yin was ordered to mount a punitive expedition against Xihe." The cycle date in the Bamboo Annals is known to be an interpolation. Nivision and Pang (1990) have successfully reproduced the erroneous cyclic date by using mathematical formulas known to pre-Quin-dynasty astronomers. With the Zuo-zhuan's citation of the Xia shu, and the Bamboo Annals' cyclic date corrected, all three eclipse records are now completely consistent with one another. We will now discuss the dating of this eclipse.

Ancient Chinese astronomers could not even forecast contemporary solar eclipses with any degree of consistency. So the only way by which they could have learned about the Zhong Kang solar eclipse is that an observational record was accurately passed down to them through the ages. Pang (1985) has successfully dated the eclipse to October 16, 1876 B.C. On that day the eclipsed Sun, visible from China, was exactly in the middle of lunar mansion Fang. Nivision and Pang (1990) have found that the Bamboo Annals chronology of the first four Xia kings - Yu to Zhong Kang - had been systematically displaced by 72 years. They have successfully reproduced this error using mathematical formulas known to pre-Qin astronomers, as discussed in the last paragraph. The corrected Bamboo Annals Xia dynasty chronology does put the fifth year of King Zhong Kang at 1876 B.C. Dating of the second Xia solar eclipse record reaffirms the accuracy of the revised Bamboo Annals chronology, which is discussed next.

\section{The Double Sunset Eclipse of 1912 B.C. and the Third Year of King Yu of the Xia Dynasty}

The recently discovered second Xia solar eclipse record is also based on three sources: (1) Philosopher Mozi (ca. 468-382 B.C.) wrote "In ancient times the san (three) Miao tribes were in disarray. Heaven ordered their destruction. The Sun rose at night..." King Yu led a punitive expedition against the san Miao, and defeated them (Condemnation of Offensive Wars III). (2) The Bamboo Annals states: "When the san Miao were about to perish...the Sun disappeared by day and reappeared at night..." (3) The Sui Chaozi account is the same as the Bamboo Annals'.

As noted earlier, a central solar eclipse occurring just before sunrise produces an optical illusion of "double dawn." Likewise such an eclipse right after sunset would be perceived as "double sunset." See descriptions of the January 4, 1992 annular sunset eclipse (Levy, 1992). Primitive people, not understanding why the sky would brighten after sunset, described the optical illusion as "sunrise at night." In ancient times night began right after sunset.

The space and time intervals for matching this eclipse are extremely tight. First, historical atlases put the san Miao domain just south of the Yangzi River, west of Lake Pengli (now Boyang) and east of Mount Heng (Herrmann, 1966). Second, the astronomically verified Bamboo Annals Xia chronology puts Yu's reign in 1914-1907 B.C. (Nivision and Pang, 1990). There was indeed such an eclipse within the eight-year and 100-mile constraints! The September 24, 1912 B.C. (Yu year 3) annular eclipse, magnitude $0.97-0.99$, produced a "double sunset" at $28^{\circ} \mathrm{N}, 114^{\circ} \mathrm{E}$-exactly over the san Miao domain! (Pang and Yau, 1996). With the accuracy of the revised Bamboo Annals early Xia chronology reaffirmed, we now calibrate the Three Dynasty royal genealogies with all 17 eclipse dates. 


\section{Discussion}

The eclipse dates, kings' names and number of generations before 841 B.C. (the earliest accurate historical date) they ruled are tabulated and plotted. We have added the Yi Zhou shu or Book of Zhou day bing-zi lunar eclipse, recorded in the 35th year of the last predynastic Zhou King Wen, and dated to january 29, 1137 B.C. (Chen, 1988).

\begin{tabular}{|c|c|c|c|}
\hline Dynasty/King & $\frac{\text { Type of }}{\text { Eclipse }}$ & $\begin{array}{l}\text { Generation } \\
\text { From } 841 \text { B.C. }\end{array}$ & Date (B.C.) \\
\hline$\overline{\text { Western Zhou } / Y i}$ & $\overline{\text { Solar }}$ & -2 & $4 / 21 / 899$ \\
\hline Western Zhou/Wen & Lunar & -9 & $1 / 29 / 1137$ \\
\hline \multirow{6}{*}{$\begin{array}{l}\text { Shang/Wu Yi } \\
\text { and Wen Ding }\end{array}$} & Solar & -10 to -11 & $10 / 31 / 1161$ \\
\hline & Solar & & $6 / 27 / 1163$ \\
\hline & Solar & & $6 / 7 / 1172$ \\
\hline & Lunar & & $7 / 2-3 / 1173$ \\
\hline & Solar & & $10 / 21 / 1198$ \\
\hline & Solar & & $5 / 6 / 1226$ \\
\hline $\begin{array}{l}\text { Shang/Lin Xin } \\
\text { and Kang Ding }\end{array}$ & Lunar & -12 & $3 / 20 / 1242$ \\
\hline \multirow[t]{6}{*}{ Shang/Wu Ding } & Lunar & -14 & $2 / 27 / 1278$ \\
\hline & Lunar & & $9 / 2 / 1279$ \\
\hline & Lunar & & $11 / 4 / 1282$ \\
\hline & Solar & & $6 / 5 / 1302$ \\
\hline & Lunar & & $11 / 24 / 1311$ \\
\hline & Lunar & & $12 / 25 / 1322$ \\
\hline Xia/Zhong Kang & Solar & -35 & $10 / 16 / 1876$ \\
\hline $\mathrm{Xia} / \mathrm{Yu}$ & Solar & -37 & $9 / 24 / 1912$ \\
\hline
\end{tabular}

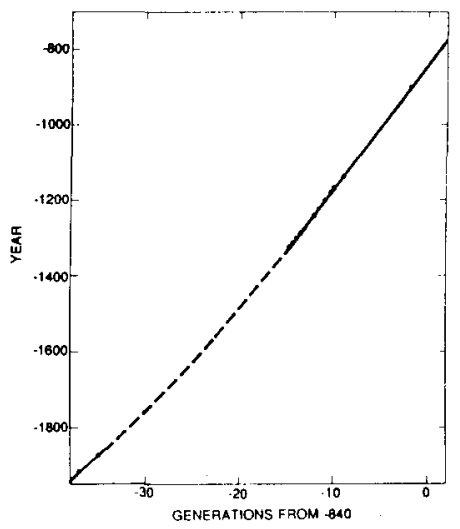

The bestfit curve above has both the strengths of verified royal genealogies - continuity - and eclipse dating - accuracy. The overall slope is -30 years/generation, as the Chinese have known all along. The slightly less steep slope for Xia is due to earlier marriage and childbearing (Mozi, Moderation in Expenditure I; Pang, 1985). The curve is $99 \%$ accurate in times with eclipse data (Western Zhou, late Shang and early Xia), but not quite as accurate elsewhere (dashed portion). We believe that our data can be confidently used as a foundation for building a detailed absolute chronology for the Three Dynasties, an important project in China's new 5-Year Plan (Song Jian, Sci. Tech. Daily, May 17, 1996; Xi, 1997; Wehrfritz, Newsweek, July 7, 1997). We thank the Dudley Observatory for financial aid.

\section{References}

Chang, Kwang-Chih (1980) Shang Civization, Yale University Press, pp. 322-329, pp. 352-355

Chen, Zungui (1988) History of Chinese Astronomy, rev. ed., Ming Wen Press, vol. 3, pp. 177-178, vol. 4, p. 42

Chou, Hung-hsiang (1958) Imperical Records of the Shang-Yin Dynasty, Privately printed in Hong Kong

Herrmann, Albert (1966) An Historical Atlas of China, Edingburgh-Aldine Publishing Co., p. 4

Hu, Houxuan (1986) Early China, Supplement No. 1, 35-38

Kiang, Tao (1981) Chinese Astronomy 5, 111-121

Levy, David H. (1992) Sky and Telescope 83, 694-695

Nivision, David S., and Pang, Kevin D. (1990) Early China 15, 87-95

Nolan, Walter I. (1975) The Facts of Keno, Gambler's Book Club, p. 17

Pang, Kevin D. (1985) paper presented at the U.S.-China Bilateral Symposium on the Analysis of Extraotdinary Flood Events, Nanjing (also Journal of Hydrology 96, 139-155)

Pang, Kevin D. (1990) Early China 15, 173-195

Pang, Kevin D., and Yau, Kevin K. (1996) in Dynamics, Ephemerides and Astronomy of the Solar System (IAU Sym. 172 Proc.), ed. S. Ferraz-Mello, B. Morando and J.-E. Arlot, Kluwer Academic Publishers, pp. 113-116

Pang, Kevin D. et al. (1989) Bulletin of American Astronomical Society 21, 753

Pang, Kevin D., Yau, Kevin, and Chou, Hung-hsiang (1995) Pure and Applied Geophysics 145, 459-485

Takashima, Ken-ichi (1988) paper presented at 40th Annual Meeting of Association for Asian Studies, San Francisco

$\mathrm{Xi}$, Zezong (1984) Archaeoastronomy 7, 34-45

$\mathrm{Xi}$, Zezong (1997) in 21st Century Chinese Astronomy, Hong Kong University Press

Yao, Xiaosui, and Xiao, Ding (1988) A General Compilation of Copies and Explanations of Oracle Bone Inscriptions from Yinsu, Zhonghua Book Publisher, p. 758

Zhang, Peiyu (1975) Acta Astronomica Sinica 16, 210-224 